Ann. Biol. anim. Bioch. Biophys., I962, 2 (I), 25-4I.

\title{
DÉVELOPPEMENT DU TESTICULE CHEZ L'AGNEAU. ÉTABLISSEMENT DE LA SPERMATOGENĖSE
}

\author{
M. COUROT \\ avec la collaboration technique de Christiane Richeris \\ Station de Recherches de Physiologie animale, \\ Centre national de Recherches zootechniques, Jouy-en-Josas (Seine-et-Oise).
}

\section{SOMMAIRE}

Dans ce travail nous avons utilisé 56 Agneaux " Ile-de-France " de I à 160 jours.

Il existe une relation étroite entre le poids dı testicule et l'état histologique des tubes séminifères : la courbe de croissance testiculaire présente deux phases distinctes - tout d'abord une phase de croissance lente, sans différenciation cellulaire, jusqu'à un poids moyen de $6 \mathrm{~g}$ - puis une phase de croissance beaucoup plus rapide, entre 6 et $200 \mathrm{~g}$ environ.

Cette seconde phase commence avec l'entrée en activité gamétogénétique des cellules germinales souches. Le rythme de la spermatogenèse y est tout de suite normal, comme le prouve la stabilité des associations cellulaires observées au sein de l'épithélium séminifère. Mais le rendement des différentes phases des processus spermatogénétiques présente d'abord une grande variabilité avant de se stabiliser autour de sa valeur théorique.

Le volume relatif occupé par les tubes séminifères dans le testicule, augmente linéairement avec l'âge ou le poids des Agneaux depuis la naissance jusqu'à la puberté. Le diamètre des tubes séminifères ne peut être relié de façon simple, ni au poids du testicule, ni à l'âge ou au poids vif des animaux pendant l'ensemble de la période considérée.

si le testicule n'est complètement développé que vers un poids de $200 \mathrm{~g}$ environ, il est déjà capable de produire des spermatozödes normaux dès qu'il atteint un poids de $65 \mathrm{~g}$.

Les travaux récents sur la spermatogenèse ont montré qu'il n'y avait pas normalement de multiplication des cellules souches chez l'adulte. A un moment précis de chaque cycle spermatogénétique, s'isole une spermatogonie souche qui, après un temps de repos, entre à son tour en activité pour donner naissance au cycle suivant

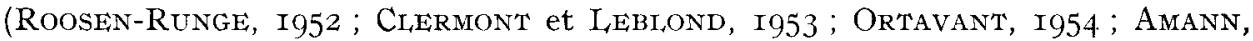
I96I).

Ainsi la capacité de production gamétogénétique du mâle adulte est déterminée, d'une part, par le nombre de cellules germinales dont il dispose au moment de la puberté, et, d'autre part, par l'efficacité des processus spermatogénétiques.

L'étude des facteurs agissant sur ces paramètres est facilitée s'il s'écoule un temps suffisant entre la naissance et le déclenchement de la spermatogenèse. Les différentes étapes des phénomènes sont alors assez isolées pour que les phases essentielles de la 
puberté puissent être mises en évidence. Ce n'est pas le cas chez les mammifères de laboratoire car la spermatogenèse y démarre très tot, quelques jours seulement après la naissance chez le Rat (RAuH, I925 ; Clermon's et PEREY, I957), le Cobaye (Bookhout, I936) ou la Souris (Hermann, I889; NeBEl, Amarose et Hacket'T, I96I). Par contre, les animaux domestiques et le Bélier en particulier semblent sur ce point un matériel intéressant.

Un certain nombre d'auteurs ont déjà étudié le développement du testicule chez cet animal, mais leur analyse a été essentiellement pondérale (WATSON, SAPSFORD et MCCANCE, I956) ou bien leurs observations histologiques peu précises ou incomplètes (Philitips et Andrews, I936; Carmon et GreEN, I952). Il était donc important de reprendte l'étude de l'établissement de la spermatogenèse chez 1'Agneau par une analyse histologique quantitative. C'est le but de ce travail.

\section{MATÉRIEL E'T MÉTHODES}

Nous avons travaillé sur lés agneaux de l'un des troupeaux de race "lle-de-France " du laborittoire. ILes testicules de 55 aninaaux ont été prélevés par castration bilatérale ou après abattage aux âges suivants : I ̀̀ $5,20,30,40,50,60,75,90,105,110,115$, I 25, I 35 à 140 et 155 à I 60 jours après la naissance. Chaque testicule était pesé après avoir été séparé de ses enveloppes, du cordon testiculaire et de l'épididyme. Puis un échantillon en était prélevé pour l'étude histologique : chez les jeunes animaux on prenait une moitié du testicule coupé suivant son grand axe et chez les agneaux plus âgés, un fragment dans l'épaisseur du parenchyme testiculaire, parallèlement à la surface de l'organe. Cet échantillon était fixé dans le liquide de Bouin-Hollande pendant 3 jours ; (quelquefois dés fragments supplémentaires étaient placés dans d'autres mélanges fixateurs, Allen B- 5 , BouinAllen, IIelly, Orth-Müller). ro à 5 minutes au maximum séparaient le prélèvement de l'organe de sat fixation. Après inclusion à la paraffine, les pièces étaient coupées à o $\mu$, colorées au Feulgen-Bleu alcian pour l'analyse histologifue.

Le volume relatif occupé par les tubes séminifères dans le parenchyme testiculaire a été mesuré suivant la technique de CHALKLEY (1943). Celle-ci, pour donner des résultats comparables, exige des coupes fixées et étalées de façon semblable dans toute la série étudiée. Notre échantillonnage basé sur too à 200 champs microscopiques par testicule a exclu ceux qui coïncidaient avec une zone du testicule comprenant une fraction de l'albuginée ou du rete testis.

Ja valeur moyenne du diamètre des tubes séminifères a été établie en mesurant sur une échelle micrométrique oculaire deux diamètres rectangulaires dans 50 sections transversales de tubes pour chaque testicule (différence maximum adnise entre deux diamètres : 1o à i 5 p. 100).

Toutes les cellules de même type ont été comptées dans 50 sections transversales de tubes prises au hasard dans chaque testicule. I.cur nombre a été corrigé suivant la méthode d'ABERCRoMibie (1946), d'après la formule :

$$
\mathrm{C}=n_{\mathrm{E}} \frac{\mathrm{L}}{\mathrm{L}+\mathrm{I}}
$$

où $n$ est le nombre de noyaux observés, $\mathrm{D}$ leur diamètre moyen mesuré à l'aide d'un microscope à projection et E l'épaisseur de la coupe. Nous n'avons pas appliqué cette formule pour corriger le nombre des spermatides âgées, ni celui des spermatozoïdes, étant donné leur forme très allongée et leur orientation dans les tubes séminifères. Nous ne l'avons pas fait non plus pour les cellules de soutien ni pour les cellules de Sertoli, compte tenu de leur forme plus ou moins irrégulière.

\section{RÉSULTATS}

\section{I. 一 ÉVOLU'IION PONDÉRALE DES TESTICULES}

Dans le tableau $x$, nous avons présenté les résultats pour chaque animal, ceux-ci étant classés par ordre croissant de poids testiculaire. Le développement pondéral des testicules ne se fait pas suivant un rythme constant : en effet, en fonction de l'âge 
TABLEAU I

Caractéristiques des testicules en rapport avec l'âge et le poids vif des agneaux "Ile-de-France"

\begin{tabular}{|c|c|c|c|c|c|}
\hline No Agneau & $\begin{array}{c}\text { Poids testicule } \\
\text { en } g\end{array}$ & $\begin{array}{l}\text { Age } \\
\text { en jours }\end{array}$ & $\begin{array}{l}\text { Poids vif } \\
\text { en } k g\end{array}$ & $\begin{array}{l}\text { Diamètre moyen } \\
\text { de } 100 \text { tubes en } \mu\end{array}$ & $\begin{array}{l}\% \text { volume } \\
\text { tubes }\end{array}$ \\
\hline 3 j. $a$ & 0,7 & 3 & - & $40 \pm 0,2 *$ & 51 \\
\hline $3 \mathrm{j}$ & 0,7 & 3 & $\ldots$ & $40 \pm 0,5$ & 46 \\
\hline 7069 & 0,8 & 't & - & $37 \pm 0,2$ & 50 \\
\hline 3409 & 0,8 & 1 & $4, t^{\prime}$ & $39 \pm 0,3$ & 48 \\
\hline 2385 & 0,8 & 1 & 5,6 & $40 \div 0,2$ & 42 \\
\hline $45: 7$ & 1,0 & 5 & 6,8 & $38 \div 0,3$ & 46 \\
\hline 141 & 1,0 & 30 & 8,2 & $36=0,2$ & $4^{\prime} t^{\prime}$ \\
\hline $14 \mathrm{~L} b$ & 1,3 & 30 & 8,1 & $41=0,2$ & 53 \\
\hline 37 & 1,1 & 19 & 11,0 & $42=0,3$ & 45 \\
\hline 112 & 1,6 & 20 & 9,0 & $19=0,2$ & 50 \\
\hline 38 & 1,6 & 18 & 7,0 & $12+0,2$ & 50 \\
\hline $1 \div 2$ & 1,9 & 31 & 11,5 & $45 \pm 0,3$ & 50 \\
\hline 113 & 2,0 & 19 & 11,0 & $46 \pm 0,7$ & 55 \\
\hline $79 \prime 5$ & 2,7 & 60 & 15,0 & $5^{\prime} t \pm 0,,_{t}^{\prime}$ & 56 \\
\hline 134 & 2,8 & 50 & 15,0 & $48=0,3$ & 57 \\
\hline 7936 & 3,1 & 43 & 11,0 & $51 \ldots 0,3$ & 52 \\
\hline 137 & 3,3 & 49 & 16,3 & $49 \leq 0,3$ & 57 \\
\hline 509 & 3,4 & 52 & 19,1 & $51 \overline{1} \quad 0,2$ & $5: 2$ \\
\hline 7925 & 3,4 & 61 & 18,0 & $52 \div 0,2$ & 57 \\
\hline 29 & 3,8 & $7 / k$ & 17,3 & $48 \doteq 0,1$ & - \\
\hline 36 & 1,2 & 74 & 20,3 & $5 \mathrm{~L}=0,3$ & - \\
\hline 8565 & 4,7 & 60 & 18,0 & $50+0,3$ & $6 i^{\prime}$ \\
\hline 7930 & 4,8 & 90 & 19,5 & 51. $\quad . . ., 3$ & 62 \\
\hline 8570 & 5,1 & 60 & 22,0 & $56 \div 0,3$ & 67 \\
\hline 136 & 5,2 & 49 & 18,7 & $51+0,3$ & 68 \\
\hline 7933 & 5,5 & 50 & 20,0 & $50 \pm 0,3$ & 51 \\
\hline 7928 & 7,6 & 90 & 21,0 & $56=0,5$ & 55 \\
\hline 818 & 7,9 & 91 & $2 / 1,5$ & $6^{\prime} \mathrm{k} \quad 0,6$ & 62 \\
\hline 7918 & 8,2 & 1.25 & 27,0 & $57 \pm 0,1$ & 60 \\
\hline 52 & 9,5 & 105 & 25,0 & $61+0,5$ & 59 \\
\hline$\geq 7$ & 11,9 & 76 & 30,3 & $61 \quad-\quad 0,,^{\prime}$ & - \\
\hline 8571 & 13,6 & 126 & 24,0 & $8^{\prime} \pm 0,8$ & 69 \\
\hline 8551 & $1 / 4,4$ & 110 & 28,0 & $66 \pm 0,5$ & 63 \\
\hline 8557 & 21,2 & 91 & 25,5 & $119 \pm 0.9$ & 69 \\
\hline 7920 & 30,7 & 105 & 29,5 & $130 \pm 0,3$ & - \\
\hline 127 & 33,0 & 117 & 29,5 & $132 \pm 1,2$ & 77 \\
\hline $85 \div 3$ & 36,2 & 110 & 38,0 & $125 \pm 1,2$ & 72 \\
\hline 7913 & 36,3 & 155 & 27,0 & $131 \pm 0,8$ & 70 \\
\hline 9733 & 50,3 & 118 & 3,0 & - & - \\
\hline 7919 & 52,0 & 125 & 33,5 & $136=0,9$ & 79 \\
\hline 7917 & 53,0 & 138 & 37,0 & 141 上 1,6 & 78 \\
\hline 9732 & 53,8 & 118 & 27,5 & - & - \\
\hline 8559 & 59,3 & 91 & 28,0 & 136 上 1,3 & 78 \\
\hline 126 & 65,0 & 117 & 36,0 & $172 \pm 1,6$ & 81. \\
\hline $85+1$ & 66,1 & 125 & 27,5 & $152 \pm 1, ' t$ & 78 \\
\hline 108 & 66,5 & 140 & 43,0 & $116 \pm 1,8$ & 75 \\
\hline 7258 & 69,8 & 136 & 37,0 & $132 \pm 1,3(\mathbf{1})$ & - \\
\hline 7915 & 70,0 & 139 & 33,0 & $146 \pm 1,2$ & 78 \\
\hline 8537 & 70,3 & 140 & 36,0 & $148 \pm 1,1$ & 80 \\
\hline 8536 & 89,9 & 140 & 39,0 & $147 \pm 1,3$ & 78 \\
\hline 815 & 98,0 & 124 & 39,7 & $163 \pm 1,9$ & 80 \\
\hline 7912 & 115,0 & 159 & 34,5 & $183 \pm 1,7\left({ }^{2}\right)$ & - \\
\hline 811 & 116,0 & 140 & 51,3 & $167 \pm 2,6$ & 81 \\
\hline 801 & 152,5 & 159 & 39,0 & $175 \neq 1,6$ & 81 \\
\hline 8503 & 165,5 & 157 & 33,0 & $201 \pm 2,6$ & 85 \\
\hline 800 & 172,5 & 159 & 46,6 & $180 \pm 3,1$ & 81 \\
\hline
\end{tabular}

* = écart type de la moyenne.

(1) $=77$ données.

$\left({ }^{2}\right)=50$ données. 


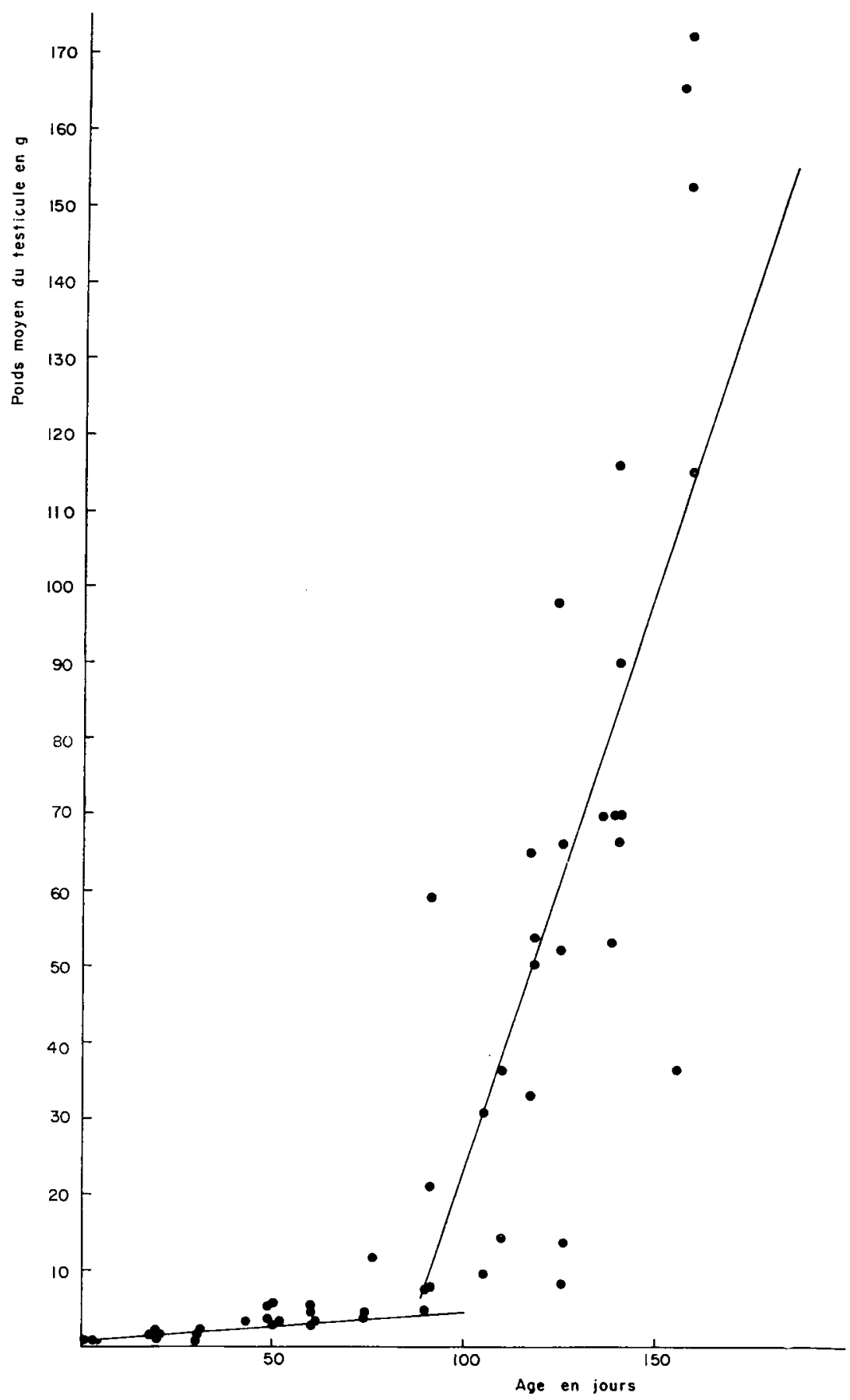

FIG. I. - Évolution pondérale du testicule en fonction de l'áge chez l'Agneau 


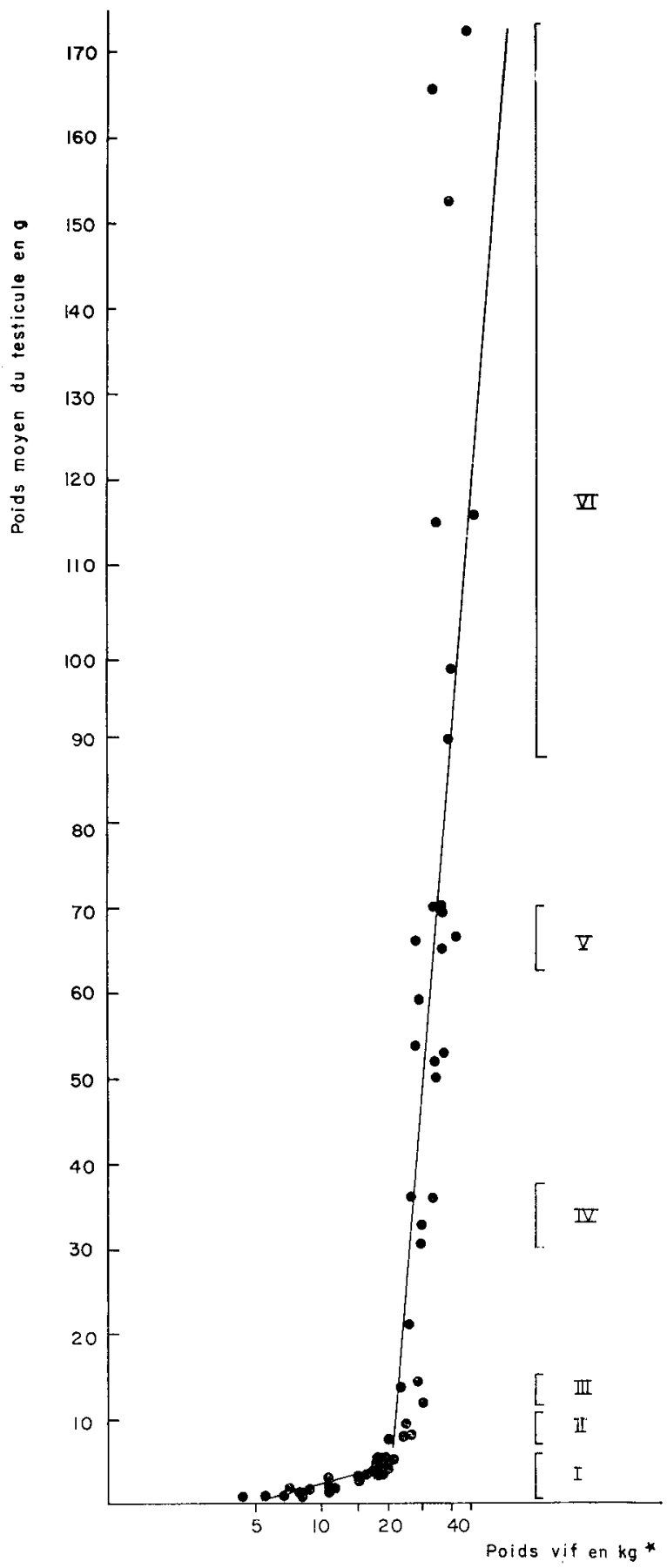

FIG. 2. - Évolution pondérale du testicule en fonction du poids vif chez l'Agneau.

* Échelle logarithmique.

I Gonocytes.

II Multiplication des spermatogonies.

III Premiers spermatocytes I.

IV Premiers spermatocytes II, puis spermatides.

V Premiers spermatozoïdes.

VI Spermatozoïdes. 
(figure I) ou du poids vif des animaux, le testicule présente schématiquement deux vitesses de croissance successives qui se traduisent en courbe par un changement de pente brutal, aux environs d'un poids de testicule de $6 \mathrm{~g}$. Ceci est particulièrement mis en évidence par l'usage d'une échelle logarithmique pour l'expression du poids vif (figure 2). Le coefficient angulaire de la courbe de croissance testiculaire par rapport au poids du corps est de $+0,27$ pour la première période et passe à $+4,9$ pour la seconde (tableau 2).

\section{TABI,EAU 2}

Corrélations et régressions entre le poids du testicule en $g(y)$ ct l'âge en jours ou le poids vif en $\mathrm{kg}(x)$ des Agneaux "lle-de-France ".

\begin{tabular}{|c|c|c|}
\hline & P'oids test//ige & I'oids test./poids vif \\
\hline & $r=+0,80(1)$ & $r=+0,93\left({ }^{1}\right)$ \\
\hline test. $<1, g r$ & $\begin{array}{l}y=0,65+0,05 x \\
n=26\end{array}$ & $\begin{array}{l}y=-0,76+0,27 x \\
n=23\end{array}$ \\
\hline test. $\gg$. & $\begin{array}{l}r=+0,75\left(^{1}\right) \\
y=-129+1,5 x \\
n=30\end{array}$ & $\begin{array}{l}r=+0,73{ }^{(1)} \\
y=-99-4,9 x \\
n=30\end{array}$ \\
\hline
\end{tabular}

Cette accélération de la croissance peut être due à une augmentation soit du tissu spermatogénétique, soit du tissu interstitiel, soit des deux à la fois. Nous avons essayé de le préciser en étudiant l'évolution de la structure même du testicule.

\section{II. - ÉVOI,UTION HISTOLOGIQUE DES TESTICULES}

L'étude histologique des testicules a porté sur trois aspects:

- importance relative des tubes séminifères dans le parenchyme testiculaire,

- variation du diamètre de ces tubes,

- étude descriptive et évolution quantitative des diffétents éléments cellulaires de l'épithélium germinal.

\section{a) Importance des tubes séminifères}

Le parenchyme testiculaire se compose de deux fractions : l'une essentiellement spermatogénétique, constituée par les tubes séminifères, et l'autre contenant à la fois le tissu interstitiel à fonction endocrine, le tissu vasculaire et le tissu conjonctif. L'importance relative des tubes séminifères est présentée dans le tableau $\mathbf{I}$. Exprimés graphiquement les résultats montrent une relation linéaires entre le volume relatif occupé par les tubes séminifères et l'âge ou le poids vifs (figure 3) des agneaux. Les corrélations et régressions sont indiquées dans le tableau 3 .

Ceci n'est évidemment valable que pour l'animal jeune. Il est certain en effet que le volume relatif des tubes séminifères ne peut pas augmenter indéfiniment avec 1'âge ou le poids des animaux : sa limite théorique supérieure est représentée par le volume qu'occuperaient des cylindres contigus dans un espace donné (voisin de go p. 


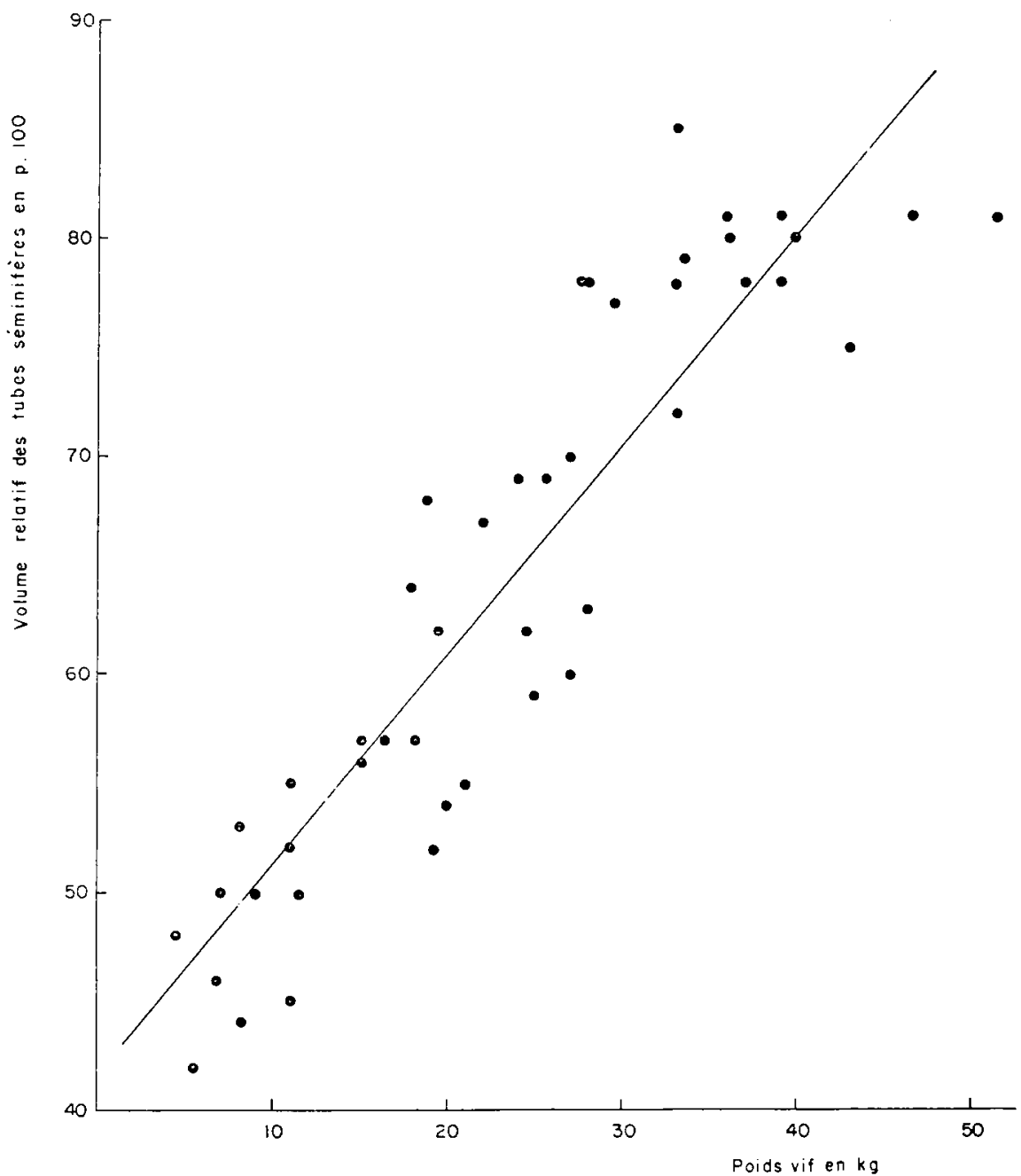

FIG. 3. - Evolution du volume relatif des tubes séminitères en fonction du poids vif chez l'Agneau $y=42+0,97 x$

$$
\begin{aligned}
& y=42 \\
& r=0,9 \mathbf{I}(\mathbf{P}<0,0,01)
\end{aligned}
$$

\section{TABLEAU 3}

Corrélations el régressions entre le volume relatif des tubes síminifères en pourcentage (y) et l'âge en jours ou le poids vif en $\mathrm{kg}(x)$ des Agneaux " Me-de-France "

\begin{tabular}{|c|c|c|}
\hline & $\%$ volume tubes âge & $\%$ volume tubes poids vif \\
\hline Coefficient de corrélation ... & $r=+0,90(1)$ & $r=+0,91\left(^{1}\right)$ \\
\hline Équation de régression .... & $y=46+0,2 \underline{2} x$ & $y=12+0,97 x$ \\
\hline Sombre de données ....... & $n=48$ & $n=45$ \\
\hline
\end{tabular}

(1) $\mathrm{P}<0,01$. 
IOo). Mais nous devons remarquer que l'envahissement du testicule par les tubes ne sera jamais aussi important puisque ceux-ci sont contournés. Si d'ailleurs nous exprimons ce volume relatif par rapport au poids moyen du testicule, nous constatons que la relation est exponentielle, elle répond à l'équation $y=47,9+16,2 x$.

Il convient donc de retenir que les tubes séminifères occupent en moyenne 50 p. Ioo du volume testiculaire chez l'Agneau, au moment de la naissance, et $80 \mathrm{p}$. Ioo environ chez l'animal pubère.

Une part essentielle de l'augmentation de poids du testicule est donc due à l'accroissement important des tubes séminifères, c'est pourquoi il est intéressant de connaître les éléments de cet accroissement.

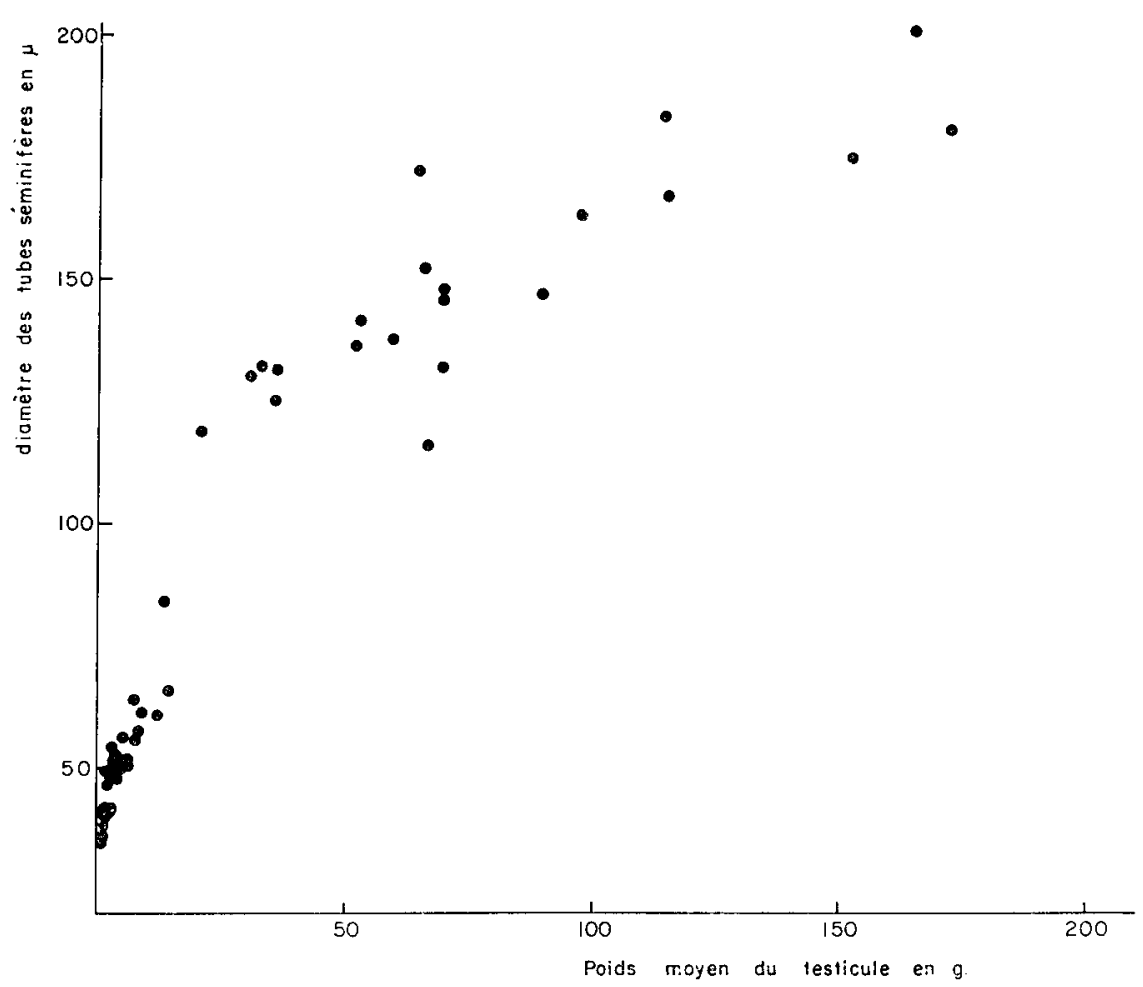

FIG. 4. - Évolution du diamètre des tubes séminifères en fonction du poids du testicule chez l'Agneau

b) Variations du diamètre des tubes séminifères

Exprimés graphiquement les résultats rapportés dans le tableau I montrent que le diamètre des tubes est en relation curviligne avec l'âge ou le poids vif des animaux ainsi qu'avec le poids moyen du testicule (figure 4). Cette relation ne répond pas à une expression mathématique simple : autour d'un poids de testicule de $\mathrm{I} 5 \mathrm{~g}$ environ il $\mathrm{y}$ a un changement assez brusque dans le taux d'augmentation du diamètre des tubes séminifères qui, à partir de ce stade, devient plus faible (figure 4). Or cette seconde 
phase correspond à l'augmentation la plus importante de la population des cellules germinales dans les tubes (tableau 5). Ce plus faible accroissement de diamètre doit donc être compensé par un allongement assez important des tubes à ce moment, comme c'est le cas dans des conditions analogues chez le Rat (CLERMONT et HUCKINS, I96I) et comme l'indique la diminution du nombre des cellules de Sertoli par coupes de tube séminifère (tableau 5).

\section{c) Évolution histologique de l'épithélium germinal}

Au cours de la vie embryonnaire il n'y a après la différenciation gonadique $\left(34^{\mathrm{e}}\right.$ jour de gestation, MAULEON, I96I) aucune transformation cellulaire importante dans les cordons sexuels et nous n'avons jamais observé de développement spermatogénétique à la naissance. Nous distinguerons au point de vue histologique deux périodes correspondant aux deux phases d'accroissement pondéral du testicule : une période que nous appellerons "gonocytaire " et une période de maturation.

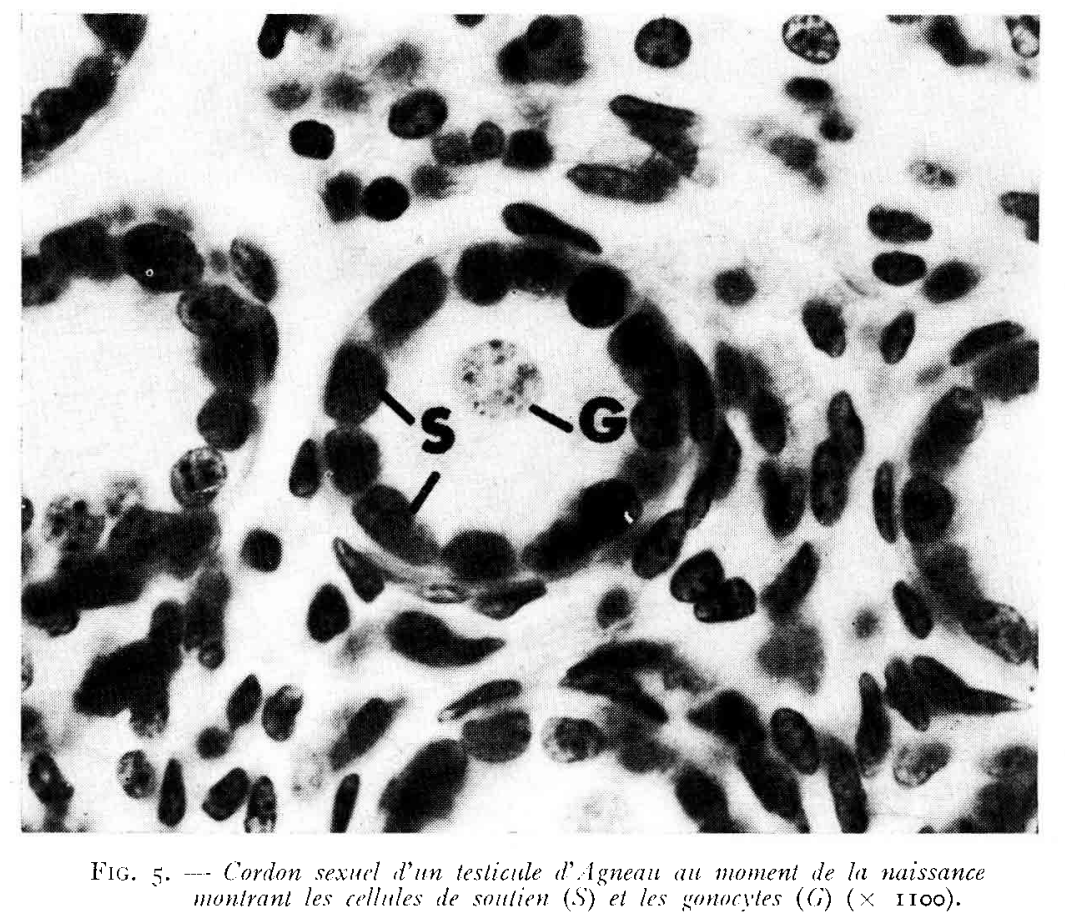

Période "gonocytaire".

A la naissance, chez l'Agneau comme dans les autres espèces (CI,ERMon'T et PEREY, I957), nous ne rencontrons que deux types de cellules dans les cordons sexuels (figure 5):

- des petites cellules assez nombreuses dont les limites sont très difficiles à discerner et dont les noyaux plus ou moins arrondis, fortement colorables, sont répartis en une seule couche le long de la membrane propre du cordon : ce sont les cellules de soutien. 
- des grandes cellules beaucoup moins nombreuses, situées au hasard à l'intérieur des cordons. Leurs noyaux sont plus grands que ceux des cellules de soutien $(7,6 \mu$ contre $5,4 \mu)$ et très réguliers ; la chromatine y présente 1'aspect d'une fine poussière faiblement colorable. Autour de la membrane nucléaire mince, on distingue assez souvent une zone cytoplasmique différenciée permettant de discerner les limites cellulaires : ce sont les cellules germinales primordiales ou gonocytes.

Contrairement aux observations de Mancini, Narbaitz et LAVIERI (i960) chez l'Homme, cet aspect des cordons sexuels reste à peu près inchangé pendant toute la période gonocytaire de l'Agneau. Seuls quelques-uns des noyaux entrent en division. Si les types cellulaires auxquels ils appartiennent sont plus difficiles à préciser, car il y a peu de différences entre "grandes " et "petites " mitoses (CLERMONT et PEREY, I957), nous avons pu cependant identifier quelques divisions de gonocytes et des divisions plus fréquentes de cellules de soutien.

\section{TABLEAU 4}

Nombre de cellules par section circulaire de tube seminifère (coupes de to $\mu$ ).

\begin{tabular}{|c|c|c|c|c|c|c|c|c|c|c|c|c|c|c|c|}
\hline \multirow{2}{*}{$\begin{array}{c}\text { No } \\
\text { Agneau }\end{array}$} & \multirow{2}{*}{$\begin{array}{l}\text { Poids } \\
\text { test. } \\
\text { en } g\end{array}$} & \multicolumn{3}{|c|}{$\begin{array}{l}\text { Cellules } \\
\text { de soutien (1) }\end{array}$} & \multicolumn{3}{|c|}{ Gonocytes $\left({ }^{2}\right)$} & \multirow{2}{*}{$\begin{array}{c}\text { No } \\
\text { Agneau }\end{array}$} & \multirow{2}{*}{$\begin{array}{l}\text { Poids } \\
\text { test. } \\
\text { en } g\end{array}$} & \multicolumn{2}{|c|}{$\begin{array}{l}\text { Cellules } \\
\text { de soutien }\end{array}$} & (1) & \multicolumn{3}{|c|}{ Gonocytes $(\mathbf{2})$} \\
\hline & & $m$ & $s_{m}$ & obs & $m$ & $s_{m}$ & obs & & & $m$ & $s_{m}$ & obs & $m$ & $s_{m}$ & obs \\
\hline $3 \mathrm{j} a$ & 0,7 & 23,1 & 上 0,3 & 100 & 0,5 & 0,04 & 100 & $79 / 5$ & 2,7 & 37,2 & $\pm 0,3$ & 100 & 1,1 & $-0,06$ & 100 \\
\hline & 0,7 & 29,4 & $\pm 0,4$ & 100 & 0,6 & 0,05 & 100 & $13{ }^{\prime}$ & 2,8 & 38,9 & 1. 0,3 & 100 & 1,1 & $\because 0$, & 100 \\
\hline 7069 & 0,8 & 33,7 & $-上 0,4$ & 100 & 0,5 & $=0,04$ & 100 & 7930 & 3,1 & 47,1 & $+0,,_{t}^{\prime}$ & 100 & 1,0 & $\therefore 0$, & 100 \\
\hline $3: 09$ & 0,8 & $3 / 4,0$ & - 0,3 & 100 & 0,6 & $-1-0,04$ & 100 & $1: 37$ & 3,3 & 41,5 & 0,6 & 100 & 1,1 & $\therefore 0$ & 100 \\
\hline 2385 & & 36,0 & $+0, k$ & 100 & 0,6 & 0,0 & 100 & 509 & & 43.7 & 0,4 & 100 & 0,9 & $=0,1$ & 100 \\
\hline 4547 & & 32,4 & 0,4 & 100 & 0,5 & 0,0 & 100 & 7925 & & $4 t^{\prime}, t$ & $+0,6$ & 100 & 0,9 & $\therefore 0$ & 100 \\
\hline $1: 1$ & 1,0 & 34,2 & $\begin{array}{ll}-1 & 0,3\end{array}$ & 100 & 0,8 & 0,0 & 100 & 29 & 3,8 & 43,6 & $+0,3$ & 100 & 1,0 & $\therefore 0$ & 100 \\
\hline $141 b$ & 1,3 & 37,8 & $\pm 0,4$ & 100 & 0,9 & $J=0,0$ & 100 & 36 & 1, & 49,8 & $\pm 0, t$ & 100 & 0,9 & $=0$, & 100 \\
\hline 37 & 1,4 & 27,3 & $\pm 0,5$ & 100 & 0,6 & $\pm 0,01$ & 100 & 8565 & 1,7 & 40,2 & $\therefore \quad 0,5$ & 100 & 0,7 & $=0,05$ & 100 \\
\hline 112 & 1,6 & 43,4 & $\therefore 0,1$ & 100 & 0,8 & $\pm=0,04$ & 100 & 7930 & 1,8 & 39,9 & I 0,5 & 100 & 0,6 & $=0,06$ & 100 \\
\hline 38 & 1,6 & 29,6 & $\pm 0,2$ & 100 & 0,4 & . & 100 & 8570 & 5,1 & 43,4 & $\pm 0,4$ & 100 & 0,7 & 0,05 & 100 \\
\hline 142 & 1,9 & 36,4 & 上 0,4 & 100 & 0,9 & . 1. $\quad 0,04$ & 100 & 136 & 5,2 & 45,1 & L. 0,5 & 100 & 1,0 & $=0,06$ & 100 \\
\hline $11: 3$ & 2,0 & 42,2 & $\because 0,4$ & 100 & 0,5 & 0,03 & 100 & 79333 & 5,5 & $3:, 6$ & $=0,3$ & 100 & 0,6 & $=0,05$ & 100 \\
\hline
\end{tabular}

( $\left.{ }^{1}\right)$ Nombre brut.

(2) Nombre corrigé.

Ces divisions assurent le peuplement des tubes séminifères dont le volume s'accroît pendant cette période puisque le poids du testicule est multiplié par 6 ou 8 , et le volume relatif occupé par les tubes augmente de io p. Ioo. Le nombre de cellules de soutien par section de tube (de ro $\mu$ d'épaisseur) passe de 30 à $40-45$ alors que le nombre de cellules germinales se maintient à peu près constant au cours de cette même période (tableau 4). Il y a donc, à l'échelon du testicule entier, une augmentation du nombre absolu des gonocytes et une augmentation beaucoup plus considérable encore du nombre des cellules de soutien.

Ceci est en rapport avec le fait que chez l'Agneau il n'y a que très peu de dégénérescences des cellules germinales contrairement à ce qui a été indiqué par d'autres auteuts chez le Rat (Clenmon't et PEREY, I957), la Souris (T'siper, I960) ou le Veau (Santamarina et Reece, I957; Abdel-Raouf, ig6o). 
Période de maturation.

a) Étude qualitative. - A partir d'un poids testiculaire voisin de $6 \mathrm{~g}$ une série de modifications interviennent au sein des tubes séminifères : la spermatogenèse s'établit.

En suivant l'évolution des deux catégories de cellules étudiées précédemment on constate que :

- Les cellules de soutien subissent une lente transformation morphologique et ne présentent presque plus de divisions. Celles-ci semblent même définitivement terminées lorsque le testicule atteint un poids de $I_{5}$ à $20 \mathrm{~g}$. C'est aussi le stade où les modifications nucléaires apparaissent plus évidentes. Les noyaux devenus moins colorables acquièrent une forme plus irrégulière avec des indentations plus ou moins profondes ; ils se placent toujours le long de la membrane propre du tube : ce sont les premières cellules de Sertoli typiques. Vers un poids testiculaire de $30 \mathrm{~g}$, presque toutes les cellules de soutien se sont transformées en cellules de Sertoli. Leur nombre relatif tend à diminuer dans les tubes (tableau 5) à la suite de l'allongement de ceuxci, on ne voit en effet pratiquement jamais de dégénérescence ou d'élimination de ce type cellulaire.

- Les cellules germinales augmentent en nombre et leur place n'est plus quelconque : elles se disposent à proximité ou même dans la couche des noyaux des cellules de soutien. En même temps on observe les stades cytologiques de transition entre gonocytes et spermatogonies souches. Mais jamais, à l'opposé de TsIPER (Ig60) chez la Souris, nous n'avons trouvé de figures de transition indiquant une transformation possible de cellules de soutien en cellules germinales.

L,es lignées spermatogénétiques commencent à se développer dans tout 1'organe; mais pour un tube séminifère donné, cela n'a pas lieu simultanément sur toute sa longueur car les différentes sections de tube n'entrent pas en activité au même moment.

On voit alors apparaître les types de spermatogonies (intermédiaires et crôitelleuses), caractéristiques du début de la lignée spermatogénétique puis, de manière très sporadique, les premières figures de prophase méiotique (figure 6) (testicule de I2 g). Le processus entamé ne s'arrêtera plus : au fur et à mesure que les lignées spermatogénétiques évoluent, d'autres entrent en activité. L'avancement de la spermatogenèse peut alors être exprimé en fonction du poids testiculaire (voir figure 2 ) : testicule de $6 \mathrm{~g}$ : début de multiplication des spermatogonies;

testicule de $x 2 \mathrm{~g}$ : premières figures de prophase méiotique;

testicule de $30 \mathrm{~g}$ : apparition des spermatocytes de second ordrespesmatides; testicule de $65 \mathrm{~g}$ : premiers spermatozoïdes libérés.

Cette libération des spermatozoïdes représente le terme des premiers cycles spermatogénétiques, mais il faut savoir que le testicule ne parvient à un stade adulte que vers un poids de $200 \mathrm{~g}$ ou plus. En effet, toutes les lignées germinales ne sont pas encore entrées en activité dans le testicule de $65 \mathrm{~g}$ et le rendement de la spermatogenèse n'est pas parvenu à sa valeur normale.

Soulignons enfin que dès le début de la spermatogenèse il existe un synchronisme dans les événements cytologiques : au fur et à mesure qu'elles se constituent et se complètent, les associations cellulaires que nous observons au sein de l'épithélium germinal sont tout à fait comparables à celles de 1'adulte (ORTAVANT, I958). Ceci avait déjà été remarqué chez le jeune Rat par CLERMONT et PEREY (I957); mais 
Nebel, Amarose et Hacke't' (I96i) le mettent en doute dans le début de l'évolution des premières spermatogonies chez la Souris.

b) Étude quantitative. - Nous avons présenté les résultats pour chacun des Agneaux étudiés dans l'ordre croissant des poids de testicule (tableau 5). Ils font ressortir un point important : exceptées les spermatogonies souches dont le nombre, par section de tube, est relativement constant lorsque le poids du testicule s'accroît, le nombre des autres cellules germinales par coupe de tube séminifère augmente avec le poids du testicule. Il se pose donc un important problème d'efficacité de la spermatogenèse dès son début.

Il est possible de suivre cette efficacité en comparant les résultats obtenus ici à ceux de la spermatogenèse du Bélier adulte (Or'TAvant, I958). Nous pouvons expri-

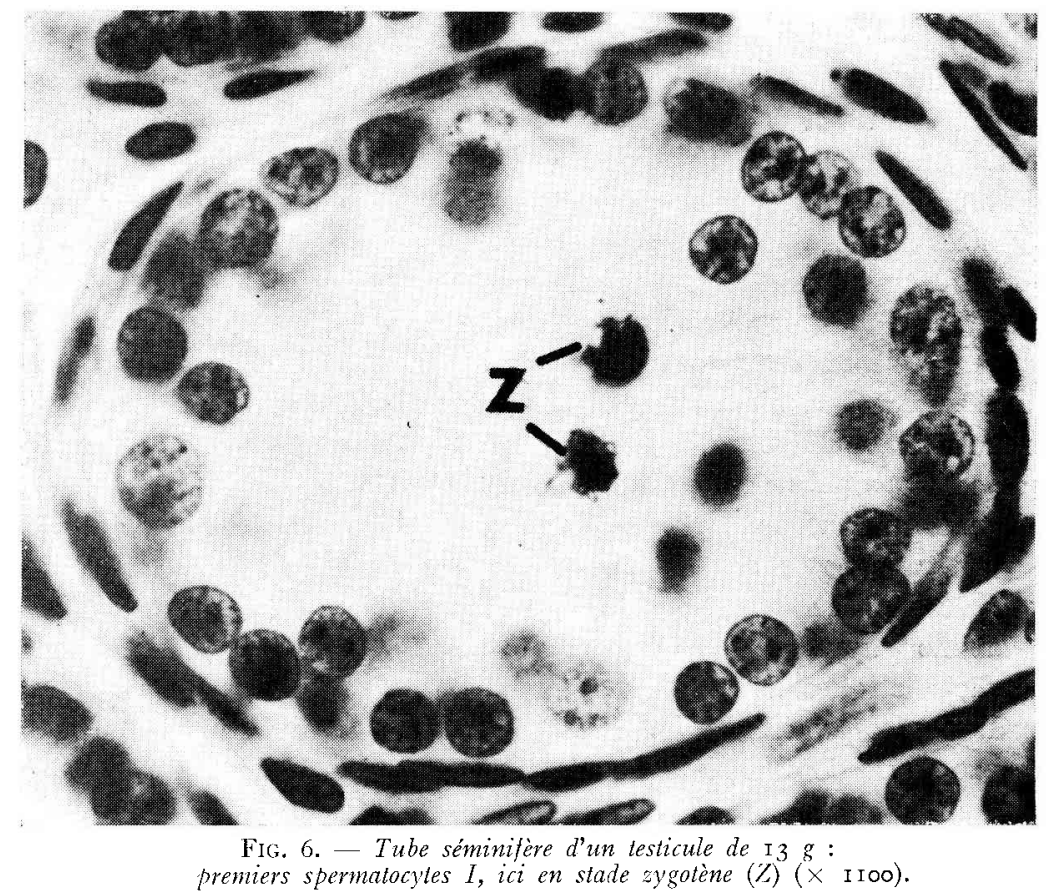

mer les rendements respectifs des divisions spermatogoniales, de la prophase méiotique et des divisions de maturation par les rapports :

I. nombre de spermatogonies souches/nombre de spermatocytes I au stade leptotène ;

2. nombre de spermatocytes I au stade leptotène/nombre de spermatocytes I à la fin du stade diplotène ;

3. nombre de spermatocytes I à la fin du stade diplotène/nombre de spermatides jeunes.

Une valeur supérieure à la moyenne théorique peut être due :

- soit à une dégénérescence cellulaire en cours d'évolution, dégénérescence qui se révèle effectivement par la présence de noyaux pycnotiques;

- soit à la présence d'un plus grand nombre de cellules souches actives dans la plus jeune génération. Ceci proviendrait de l'entrée en activité de nouvelles sperma- 

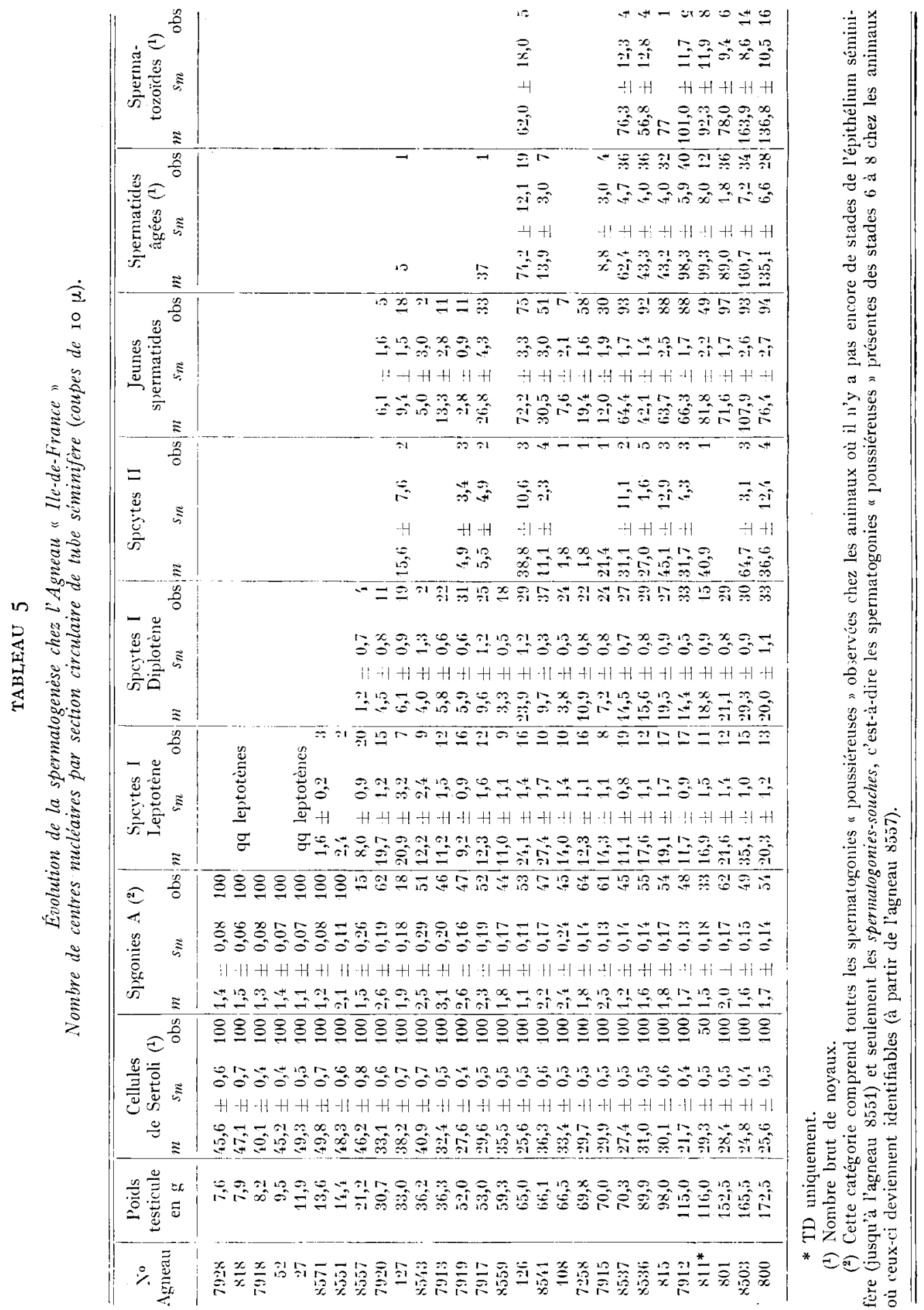
togonies souches à côté des spermatogonies de renouvellement des lignées germinales déjà en évolution, et aurait lieu jusqu'à ce que toutes les lignées germinales ou à peu près, soient en jeu.

Les valeurs des rapports que nous avons définis présentent au début une grande variabilité (fig. 7) et pratiquement ce n'est qu'à partir d'un poids testiculaire de Ioo $g$ que les valeurs trouvées ne diffèrent plus significativement des valeurs théoriques de l'adulte (tablean 6).

TABLEAU 6

Rapport du nombre des cellules dans l'épithélium germinal de l'Agneau

\begin{tabular}{|c|c|c|c|c|}
\hline No Agneau & $\frac{\text { Poids testicule }}{\text { en } g}$ & $\frac{\text { Gonies A }}{\text { Cytes I leptot. }}$ & $\frac{\text { Cytes I leptot. }}{\text { Cytes I diplot. }}$ & $\frac{\text { Cytes I diplot. }}{\text { Jeunes spides }}$ \\
\hline 8557 & 21,2 & 0,19 & 6,67 & \\
\hline 7920 & 30,7 & 0,13 & 4,28 & 0,75 \\
\hline 127 & 33,0 & $0,09(1)$ & 3,49 & 0,65 \\
\hline 8543 & 36,2 & 0,20 & 3,05 & 0,80 \\
\hline 7913 & 36,3 & 0,28 & 1,93 & 0.44 \\
\hline 7919 & 52,0 & 0,28 & 1,56 & 2,11 \\
\hline 7917 & 53,0 & 0,19 & 1,31 & 0,35 \\
\hline 8559 & 59,3 & 0,16 & 3,33 & \\
\hline 126 & 65,0 & 0,05 & $1,05\left({ }^{1}\right)$ & $0,3 \ddot{2}$ \\
\hline 8541 & 66,1 & $0,08 \quad(1)$ & $2,8:$ & $0,3 \ddot{2}$ \\
\hline 108 & 66,5 & 0,17 & 3,68 & 0,49 \\
\hline 7258 & 69,8 & 0,15 & $1,1^{\prime}+\left({ }^{1}\right)$ & $0,5 i$ \\
\hline 7915 & 70,0 & 0,17 & 1,99 & 0,61 \\
\hline 8537 & 70,3 & $0,11 \quad(1)$ & 0,77 & $0,23(1)$ \\
\hline 8536 & 89,9 & $0,09(1)$ & $1,13 \quad(1)$ & 0,37 \\
\hline 815 & 98,0 & $0,09(1)$ & $0,98(1)$ & 0,31 \\
\hline 7912 & 115,0 & 0,15 & $0,81 \quad(1)$ & $0,2 \geq 2$ \\
\hline 811 & 116,0 & $0,09(1)$ & $0,90(1)$ & $0,28(1)$ \\
\hline 801 & 152,5 & $0,10(1)$ & $1,03\left(^{(1)}\right.$ & 0,29 \\
\hline 8503 & 165,5 & $0,05(1)$ & 1,20 & $0,27(1)$ \\
\hline 800 & 172,5 & $0,08\left(^{1}\right)$ & $1,02(1)$ & $0,26(1)$ \\
\hline \multicolumn{2}{|c|}{ Rapport théorique chez l'adulte } & $1 / 16=0,0625$ & 1,00 & $1 / 4=0,25$ \\
\hline
\end{tabular}

(1) Tas significativement différent de la valeur théorique.

\section{CONCLUSION}

Cette analyse de 1'établissement de la spermatogenèse chez 1'Agneau permet de dégager plusieurs données.

Entre la naissance et l'état adulte, la croissance testiculaire d'abord lente pendant la période gonocytaire s'accélère pendant la période de maturation du testicule. Cette évolution pondérale est plus en liaison avec le poids corporel qu'avec l'âge des animaux pendant la première période (tableau 2 ). Cela indique que le démarrage de la spermatogenèse, quii commence après cette phase, dépend d'un certain état de développement de l'animal plus que de son âge. Lorsque cette accélération de croissance du testicule intervient, c'est essentiellement sous l'effet de l'établissement de 
la fonction spermatogénétique caractérisée par une multiplication cellulaire de plus en plus intense et par l'augmentation de l'importance des tubes séminifères (taille et proportions relative).

Nous observons chez l'Agneau la même filiation cellulaire que celle qui est rencontrée chez le Rat (CLERMont et PEREY, I957). Ce sont les gonocytes et uniquement

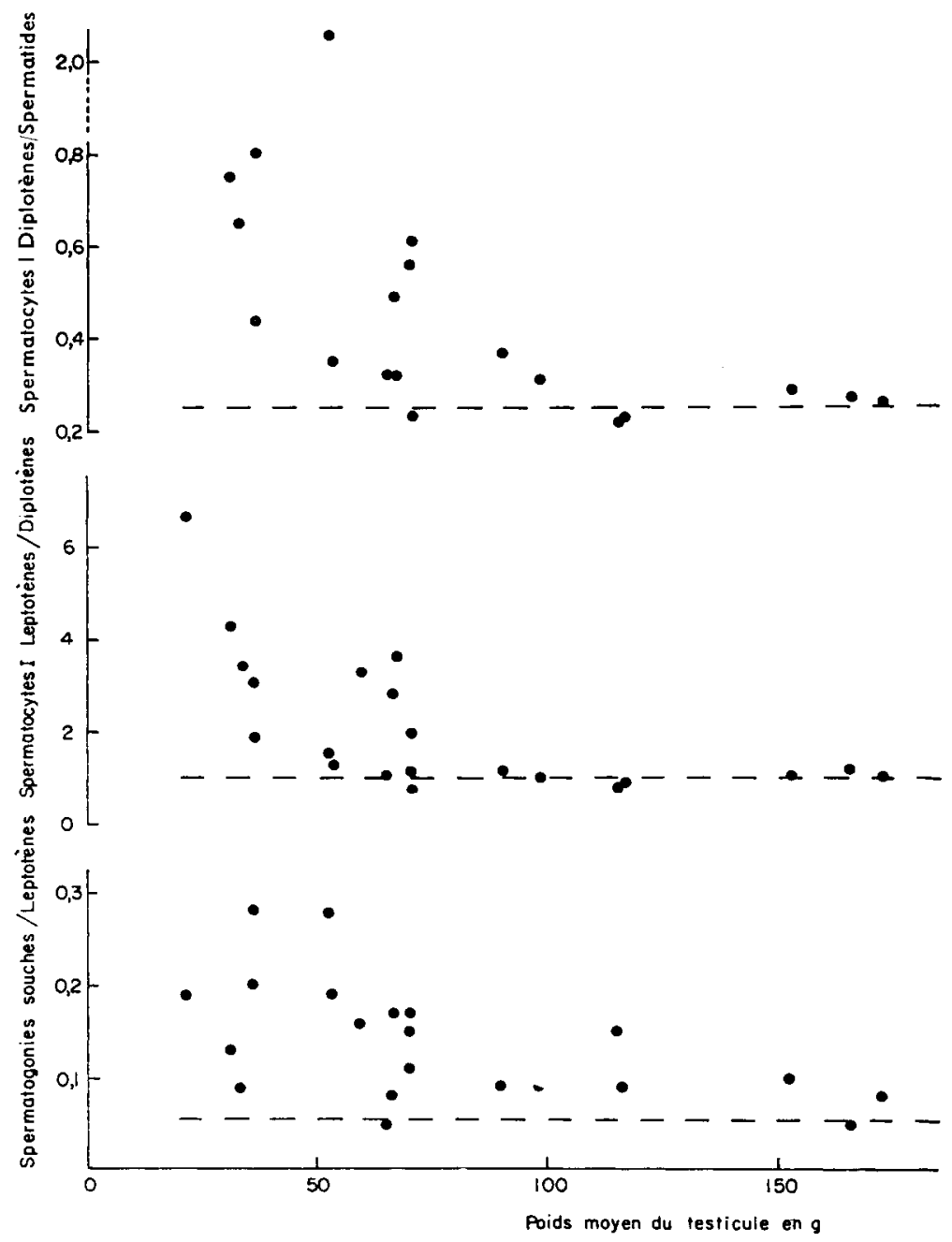

Fig. 7. - Évolution de l'activité spermatogénétique en fonction du poids du testicule chez l'Agneau

eux qui donnent les cellules germinales adultes, alors que les cellules de Sertoli ne proviennent que des cellules de soutien. Par ailleurs, dès le début de l'établissement de la spermatogenèse, nous trouvons, plus ou moins complètes, les mêmes associations cellulaires que celles observées chez l'adulte. Cette identité avec l'adulte est la preuve certaine que dès l'instant où elle s'établit, la spermatogenèse le fait suivant son rythme propre définitif. 
L'évolution du rendement de la spermatogenèse montre que celui-ci n'est normal qu'au bout d'un certain temps. C'est donc sous cet aspect quantitatif, plutôt que qualitatif, qu'il faut envisager les phénomènes de l'établissement de la spermatogenèse.

Ręu en ncvembre 1961

\section{SUMMARY}

The development of the testis of tile lamb. Cytological study

The weight and the histology of the testes were observed on 56 "Ile-de-France "lambs between $I$ and I60 days old.

The growth curve of the testis shows a clear change at a weight of about $6 \mathrm{~g}$. Before this level the testis has an infantile histological appearance with only slightly active sex cords in which one can observe two types of cells : the gonocytes and the supporting cells.

This change in weight can be correlated with the beginning of spermatogenesis, and afterwards, there is a close relationship bet ween the development of spermatogenesis and the weight of the testis:

Testis weight $6 \mathrm{~g}$ : multiplication of type " $\mathrm{A}$ " spermatogonia.

I $2 \mathrm{~g}$ : appearance of primary spermatocytes.

$30 \mathrm{~g}$ : appearance of spermatids.

$65 \mathrm{~g}$ : appearance of spermatozoa.

$200 \mathrm{~g}$ : adult testis.

The cellular associations seen in the germinal epithelium during this phase are the same as those in the adult testis. This indicates that a normal rhythm of spermatogenesis is established right from the beginning.

The ratios of the various types of cells indicate that there are quantitative differences in the first spermatogenic waves wich rapidly stabilize to about the normal value for the adult.

The relative volume of the seminiferous tubules in the testis is in a linear relationship with the age $(r=0,90)$ and with the body weight $(r=0,9 \mathrm{I})$ of the animals from birth to puberty. There is no simple relationship between the diameter of the seminiferous tubules and the testis weight.

\section{RÉFÉRENCES BIBLIOGRAPHIQUES}

ABIEL-RaOUF M., 1960. 'The postnatal development of the reproductive organs in bulls with special reference to puberty. Acta Endocr., 34, sup. 49, Io9 pp.

Abercrombie M., 1946. Estimation of nuclear population from microtone sections. Anat. Rec., 94$238-248$.

Amanv R. P., rg6r. Reproductive physiology of the Male Bovine. Ph. D hesis, the Pennsylvania State University University Park. $206 \mathrm{pp}$.

Booknout C. G., I936. The gern cell cycle in the gruinea pig. II. The postnatal development of the testis Z. Zellforsch., 25, 749- 763 .

Carmon J. L., Grenn W. W., I952. Histological study of the development of the testis of the ram. J. Anim. Sci., 11, 674-687.

Chalkley H. W., 1943. Nethod for the quantitative morphologic analysis of tissues. J. Nat. Canc. Inst. 4, 47-53.

Clermont Y., Huckins (., I $96 x$. Mirroscopic anatomy of the sex cords and seminiferous tubules in growing and adult male Albino rats. Am. J. Anat., 108, $79-97$.

Ciermont Y., Leblond C. P., i953. Renewal of spermatogronia in the rat. Am. J. Anal., 93, 475-502.

Clermont Y., Perey B., i957. Quantitative study of the cell population of the seminiferous tubules in immature rats. Am. J. Anal., 100, 24r-267.

HermanN F., r889. Die postfoetale Histiogenese des Hodens der Maus bis zur Pubertät. Archiv mikr. Anat., 34, 429-437.

Mancini R. E., Farbaitz R., Lavieri J. C., r960. Origin and development of the gemmative epithelium and sertoli cells in the human testis : cytological, cytochemical and quantitative study. Anat. Rec., 136, $477-490$. 
Mauleon l'., ig6r. Déroulement de l'ovorenèse comparé chez différents mammifères domestiques. Proc. IVih Int. Congr. Anim. Reprod. The Hague. Yo K.I. I52.

Neber B. R., Amarose A. P., Hackett F. M., ig6r. Calendar of gametogenic development in the prepubertal male mouse. Science, 134, $832-833$.

Ortavant R., 1954. Etude des générations spermatogoniales thez le Bélier. C. R. Soc. biol., 148, I958Ig6o.

Ortavant R., I958. Le cycle spermatogénétique chez le Bélier. Thèe Doct. Paris, 127 pp. ed. 1959, Inn. Zootech., 8, 183-24t et $271-322$.

Pinllips R. W., Andrews F. N., I936. The development of the testes and scrotum of the ram, bull and boar. Mass. Agric. Exp. Stat. bull., 381, 16 pp.

Ravir W., 1925. Ursprung der männlichen Keimzellen und die chromatischen Vorgänge bis zum Entwickelung der spermatocyten. Z. Anat., 76, 56r-577.

Roosen-Runge F. C., 1952. Kinetics of spermatogenesis in mammals. Ann. Nere York Acad. Sci., 55, $574-584$.

Santamarina E., REece R. P., i957. Normal development of the germinal epitelium and seminiferous tubules in the bull. Im. J. Vet. Res., 18, 261-278.

Tsiper S. M., 1960. Origin of spermatogonia in albino mouse. Zh. Obshei. Biol., 21, 20-27.

IVATSON R. II., SAPSFORD C. S., MCCANCE I., 1956. The development of the testis, epididymis and penis in the young merino ram. Alstr. J. Agric. Res., 7, 57t-590. 